\title{
Characterizing Polygonality in Biological Structures
}

\author{
Luciano da Fontoura Costa \\ Instituto de Física de São Carlos. Universidade of São Paulo, \\ São Carlos, SP, PO Box 369, 13560-970, phone +55 163373 9858, \\ FAX +551633713616, Brazil, luciano@if.sc.usp.br
}

\author{
Fernando Rocha and Silene Maria Araújo de Lima \\ Centro de Ciências Biológicas, \\ Departamento de Fisiologia, \\ Universidade Federal do Pará, \\ Campus Universitário do Guamá, \\ Rua Augusto Corrêa 01, \\ CEP 66075-000, Belém, Pará, \\ Brazil
}

(Dated: 9th Oct 2005)

\begin{abstract}
Several systems involve spatial arrangements of elements such as molecules or cells, the characterization of which bears important implications to biological and physical investigations. Traditional approaches to quantify spatial order and regularity have relied on nearest neighbor distances or the number of sides of cells. The current work shows that superior features can be achieved by considering angular regularity. Voronoi tessellations are obtained for each basic element and the angular regularity is then estimated from the differences between the angles defined by adjacent cells and a reference angle. In case this angle is 60 degrees, the measurement quantifies the hexagonality of the system. Other reference angles can be considered in order to quantify other types of spatial symmetries. The performance of the angular regularity is compared with other measurements including the conformity ratio (based on nearest neighbor distances) and the number of sides of the cells, confirming its improved sensitivity and discrimination power. The superior performance of the haxagonality measurement is illustrated also with respect to a real application concerning the characterization of retinal mosaics.

PACS numbers: 05.50.+q, 64.60.Cn, 87.80.Vt, 05.65.+b
\end{abstract}

\section{INTRODUCTION}

Several important properties of biological systems are directly related and even determined by the spatial distribution of their constituent elements. For instance, the distribution of ganglion cells through the mammals retina is known to accurately sample the visual field with just the right amount of overlap. Similarly, the adjacency of cells is important for cell signaling, while the spatial distribution of cells actively expressing some specific gene is immediately related to tissue and organ formation. Other examples where the spatial distribution of the elements is critical involve the spread of pathological agents such as viruses and bacteria as well as the spatial arrangement of diseased cells and lesions.

Typically, spatial order in biological systems involves structured/symmetric arrangements of points such as in hexagonal systems. These structures can be understood as regular lattices, characterized by fixed spacing and angles between the constituent points. Even in the cases where the boundaries between the elements are not available, they can be obtained from the Voronoi tessellation considering the original points as seeds (e.g. 12]). The ubiquity of polygonal organization in biological systems is related to special packing and physical properties allowed by ordered systems (e.g. [17]). For instance, it is known that the hexagon is the regular polygon with max- imum number of sides which can be used to tile the plane. The immediate advantage of such a regular tiling is the maximization of the number of neighbors of each element, with immediate implications for cell signaling [8], resolution and isotropy in sensory acquisition (e.g. photoreceptors, retinal neurons and omatidea), structural properties (e.g. choral, cell membrane, striate muscle fibers, and other structures), to name but a few cases. Interesting combinations of pentagons and hexagons are also often found in three-dimensional biological structures such as pollen, viruses and radiolaria structures.

Because spatial regularity plays such a key role in defining specific structural and functional properties of biological systems at micro, meso and macroscopic spatial scales, it becomes of paramount importance to quantify in an objective and accurate way the degree of spatial organization of such systems. Among the previous works aimed at automated quantification of spatial distributions, the most frequently adopted approach involves counting the number of sides of each cell [11, 14]. Another traditional approach which can be used to quantify polygonality involves the use of the distance between nearest neighboring points as well as derived measurement such as the conformity ratio (e.g. 3, 15, 16]). A comparison of methods for characterization of spatial properties of retinal mosaics has been presented by Cook [3], with emphasis on nearest neighbor distances. 
The present article starts by identifying the main properties required from a good measurement of spatial order and follows by describing each of the considered measurements, whose properties are then evaluated for the characterization of global and local spatial order. The analysis of the potential of the measurements to characterized global properties takes into account simulated (hexagonal lattices with progressive perturbations) as well as real data (retinal mosaics of photoreceptors). The possibility to use the measurements to quantify local order around each point is also addressed with respect to the identification of regions with distinct spatial order and the analysis of a system involving progressive (radial) variation of spatial order.

\section{REQUIREMENTS}

Before considering possible indicators of spatial order and evaluating their performance, it is important to identify the main features which could be expected from a good such measurement. Such features are discussed in the following.

Let us consider that the property $P$ to be measured is a function of a given parameter $s$ 18], so that $P=P(s)$, and that the act of measuring $P$ involves mapping it into the quantity $Q(P(s))=Q(s)$. For instance, $s$ can be the intensity of spatial perturbation added to a perfectly hexagonal lattice (see Section IVA), and $Q$ is the measurement of spatial order. Note that typically we do not have access to the values of $P$, otherwise there would be no need to consider its measurement. Figure 1illustrates mappings from the property $P$ into two possible measurements $Q(s)$. Although this example assumes that $P$ is upper and lower bound, some situations may imply unlimited values of $P_{\min }$ or $P_{\max }$. Situations such as that depicted in Figure 1 can be always normalized, by shifting the function along the $x$ - and/or $y$-axes, such that $\tilde{P}_{\min }=\tilde{Q}_{\min }=0$ and $\tilde{P}_{\max }=\tilde{Q}_{\max }=1$, defining a linear relationship. The mapping in (b) can also be linearized by using an additional transformation of the curve itself.

Sensitivity/Linearity: One important feature of any measurement is its ability to respond to variations of the measured quantity. Because measurements can be normalized, sensitivity should be considered in relative terms along the interval of measurement of interest. For instance, the mapping in Figure 1(a) is more sensitive than that in (b) for small values of $P(s)$, but becomes less sensitive for large values of $P(s)$. The sensitivity of the measurement in Figure 11(a) is the same for any value of $P(s)$. Observe that the increase of sensitivity along one of the regions of the mapping can only be achieved at the expense of loss of sensitivity at another region. Unless some specific interest of achieving enhanced sensitivity at some specific range of values is to be considered, measurements should ideally be linear. In the specific case of spatial order quantification, one is frequently interested

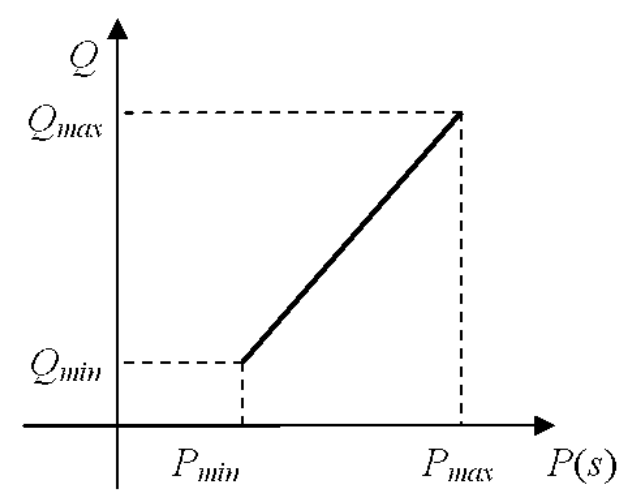

(a)

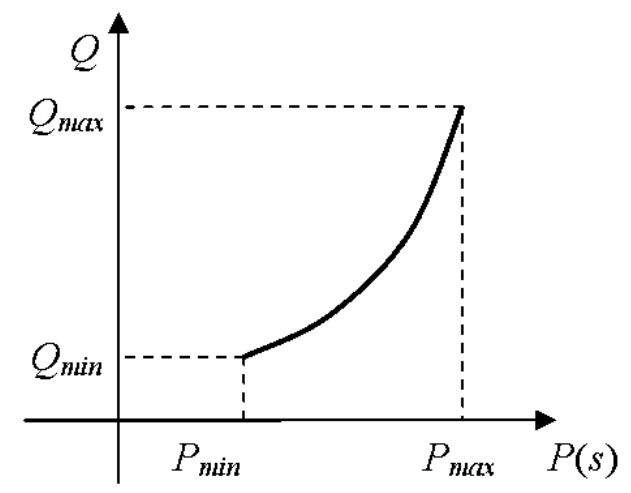

(b)

FIG. 1: Two possible measurements mapping the property $P$ into $Q$.

in the cases of small deviations from perfect order. In such cases, it is interesting to obtain greater sensitivity for higher values of spatial order.

Generality, Uniformity and Invariance: It is usually interesting that the measurement of $P$ be monotonic (increasing or decreasing), well-defined and with uniform properties along the whole interval of measurements. This implies absence of singularities (e.g. tendency to go to infinity for some values of $P$ ) as well as uniform sensitivity. The linear mapping meets all such requirements. In cases such as the quantification of spatial order of geometrical systems, it is also important that the measurements be invariant to translation, rotation and scaling.

Discriminative Power: Although Figure 1 considered a deterministic mapping from $P$ into $Q$, it often happens that measurements taken over different realizations of a system for the same parameter $s$ yield different values. This is often the case with spatial order. For instance, the spatial order of the photoreceptors in the retina of two similar animals (i.e. same age, gender, etc.) from the same species will unavoidably be characterized by distinct, even though similar, values. In turn, such a stochastic variation of $Q$ implies that in certain situa- 
tions two realizations of a system, respective to different parameters $s$, will yield the same measurement value. In other words, the stochastic variability of $Q$ will tend to limit its discriminative power. Given two instances of $s$, i.e. $s_{1}$ and $s_{2}$, the discriminative power will vary inversely with the overlap between the statistical density functions characterizing $P\left(s_{1}\right)$ and $P\left(s_{2}\right)$. Such an overlap can be estimated by considering the average and standard deviation of those two densities, which is the procedure adopted in the present article.

Adimensionality: While this is not an imperative property, it is interesting that the measurement in question be adimensional, allowing it to be expressed in percentage values. In addition, adimensionality often facilitates multivariate statistical analysis and testing (e.g. 9, 10]).

\section{METHODS}

Let the image containing the biological system under analysis be represented in an $N_{i} \times N_{j}$ image $A$. Each element of the system is identified by an integer label $i$, while its spatial position is indicated in terms of the coordinates $(x(i), y(i))$ of some reference point or seed, often corresponding to the centroid of the elements (see Figure 2a). The nearest neighbor distance between a point $i$ and a set of points $S$ is defined as the smallest distance between $i$ and each of the points in $S$. The Voronoi tessellation of the system of reference points can be obtained by assigning to each pixel $A(i, j)$ the label of the nearest reference point. One of the nice features of Voronoi tessellations is that they can be used to establish boundaries and adjacencies between the original reference points. More specifically, given a reference point $k$, each of its Voronoi neighbors will correspond to a reference point $i$ such that the Voronoi cells of $k$ and $i$ share a common side. In order to avoid border effects, the Voronoi cells which are adjacent to the image background (i.e. the border cells) are henceforth identified and excluded from the calculations.

Number of Voronoi cells: An interesting measurement which can be immediately obtained from Voronoi tessellations corresponds to the number of sides of each Voronoi cell, a measurement which has been called hexagonality (e.g. 11, 14]). Henceforth $n_{k}$ represents the number of sides of the cell associated to the reference point $k$. An hexagonal system, for example, will produce all cells with 6 sides, except for the cells at the border of the system.

Angular Regularity: For each Voronoi cell $k$, starting at an arbitrary neighbor $i$, determine the successive $N_{k}$ neighbors as one turns around the cell in a clockwise fashion and calculates the angles $\alpha_{i}$ defined by the adjacencies (see Figure 2a). The angular polygonality of a given point $k$ can then be expressed in terms of the sum of angle differences for each point, i.e.

$$
\Sigma=\sum_{i=1}^{N_{k}}\left|\alpha_{i}-\beta\right|
$$

where $\beta$ is a specific angle of interest. In case one is interested to quantify the angular hexagonality of a point system, $\beta$ should be taken as 60 degrees. Similarly, in case one wants to check for orthogonal arrangement, $\beta$ should be 90 degrees. Other values of $\beta$ varying in the interval $0<\beta<180^{\circ}$ can also be used in order to check for other types of angular distributions. However, only 60, 90 and 120 degrees allow full regular tiling of the plan. Figure 3 shows the polygonalities of a single point surrounded by $N$ points (uniform angle spacing). Note that the sum of angle differences varies from 0 to infinite (i.e. a point surround by an infinite number of points). Although it would be possible to consider the average of angle differences (i.e. divide the sum of angle differences in Equation 1by the number of neighbors of each point), such an alternative would lead to similar measurements as the number of neighboring cells increase, therefore reducing the discriminative power.

An alternative measurement of spatial order, yielding the adimensional measurement henceforth called the polygonality index, can be defined as a function of the sum of difference angle as follows

$$
\Delta_{\alpha}(k)=\frac{1}{\sum_{i=1}^{N_{k}}\left|\alpha_{i}-\beta\right|+1}
$$

This measurement, which was first considered for hexagonality characterization in [4], is bound between 0 (lack of spatial order) to 1 (perfect polygonality).

Conformity Ratios: Once all nearest neighbor distances have been calculated, for instance by using the simple and effective algorithm described in [3], the conformity ratio of distances can be defined [16] as the ratio between the mean and standard deviation of all nearest neighbor distances. More organized systems should therefore imply higher conformity ratios.

Coefficient of Variation: The exact inverse of the conformity ratio, the coefficient of variation is also considered in this work because of its traditional use in statistics.

Note that the conformity ratio of the nearest distances present a singularity (tends to infinity) when the shortest distances for every point in the system is equal, as with regular lattices. The coefficient of variation does not present singularities, but will produce null values for any regular lattice (null standard deviation of shortest distance will imply coefficient of variation equal to zero).

Although it would be possible to define conformity ratio and coefficient of variation of angles differences, such measurements would be completely unable to cope with situations such as that considered in Figure 3 i.e. involving $N$ equal (or similar) angles around a point. In these 


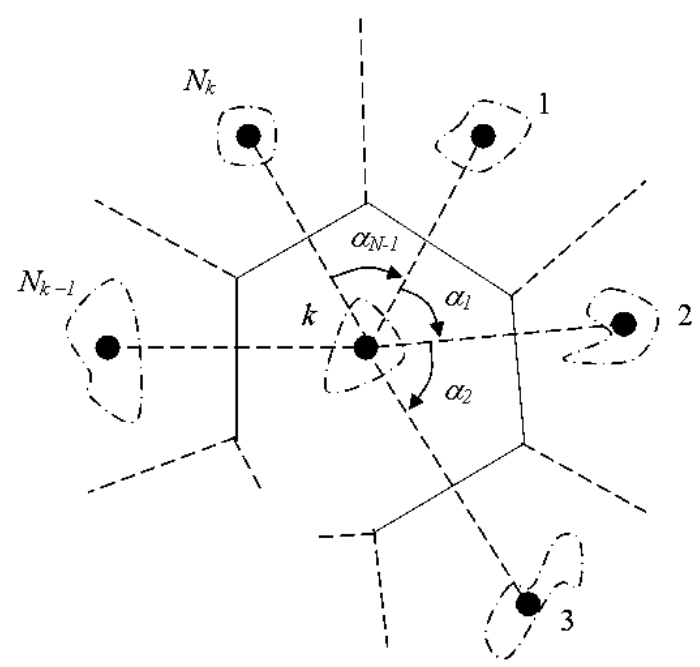

(a)

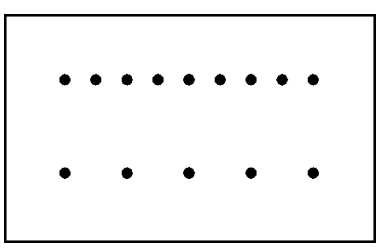

(b)

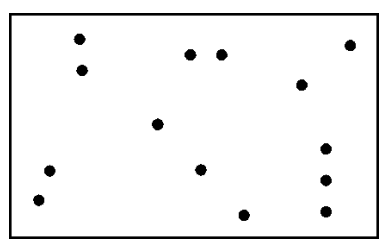

(c)
FIG. 2: The geometrical construction and symbols used in the definition of the angular hexagonality are presented in (a). The reference points are shown as black dots, and the neighbors of seed $k$ are enumerated in clockwise fashion from 1 to $N_{k}$. Although rather distinct, the two point distributions in (b) and (c) yield identical statistics of shortest distances, and hence the same conformity ratio.

cases, the standard deviation of the angle values is null, implying conformity ratio equal to zero and coefficient of variation equal to infinity. Such a property also implies lack of sensitivity for systems characterized by a high level of spatial order. Contrariwise, the sum of angle differences or hexagonality index will produce distinct values for any number of equally spaced neighboring points. For such reasons, and also because of poor performance in preliminary experimental investigations conducted by the authors, the coefficient of variation and conformity ratio of angle differences will not be considered further in this article.

Although all the above measurements are invariant to translation, rotation and scaling of the system under analysis, any measurement derived from the minimum distance statistics will fail to distinguish between situations such as those illustrated in Figure 2(b) and (c), which yield identical statistical densities of shortest distances and therefore equal conformity ratios. This important limitation of the shortest distance measurement stems from the fact that it only takes into account the most immediate neighborhood around each point, over-

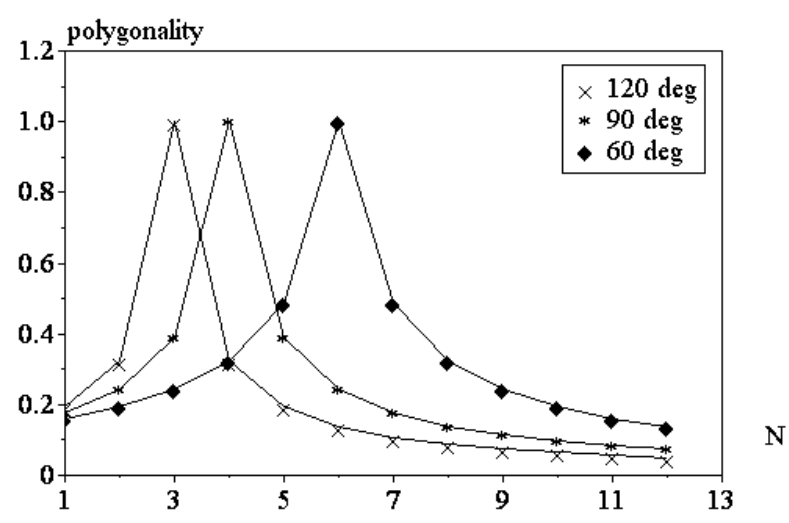

FIG. 3: The poligonalities for $\beta=120,90$ and 60 degrees obtained for a single point surrounded by $N$ uniformly distributed angles (i.e. $\alpha=2 \pi / N)$. Observe that the sharp peaks clearly identify each type of symmetry. For instance, the perfect hexagonal arrangement of neighbors for $N=6$ implies the sharp peak of the poligonality assuming $\beta=60$ degrees at that respective position.

looking spatial properties at higher spatial scales. Therefore, a natural means to try to obtain better discriminative power while using distance statistics consists in considering successive shortest distances, such as the second, third, etc., shortest distances. Such a possibility is also explored in this work.

A severe limitation of the method of counting the number of Voronoi cell sides follows from the fact that the number of cell sides will not change except for gross perturbations of the particle system. However, this measurement can be potentially useful when the structures under analysis involve large perturbations.

\section{RESULTS}

This section describes a systematic performance comparison of methods used to quantify the spatial order of point distributions, including nearest distances, number of Voronoi sides, conformity ratio and coefficient of variation of the nearest distance, sum of angle differences and hexagonality index. This evaluation considers the quantification of global and local spatial order in systems of points. While the former case addresses the problem of assigning a single measurement to the whole system of points, the latter situation involves the quantification of the spatial order around each point of the system. The global evaluation is performed for simulated data (regular hexagonal lattices with progressive spatial perturbations) and real data concerning agouti (Dasyprocta agout) retinal photoreceptor mosaics. The obtained results clearly corroborate the superiority of the sum of difference angles and the hexagonality index, especially regarding their linearity, sensitivity and discriminative power. The investigation of the potential of the considered measurements 
for quantifying local spatial order involves the identification of regions with different levels of order as well as a system where the spatial order varies radialy.

\section{A. Simulated Data}

In order to investigate the performance of the above measurements under a controlled situation, hexagonal lattices with progressive spatial perturbation intensities were obtained by using mathematic-computational means. More especifically, hexagonal lattice with 621 points and vertices of length $\Delta=10$ pixels were generated in a $480 \times 480$ pixels image and progressively perturbated with uniformly distributed displacements of magnitude $\delta=1,2, \ldots, 10$. A total of 50 realizations of each configuration (i.e. each perturbation intensity) was performed in order to enhance statistical representativity. Figure 4 shows two of the considered simulated hexagonal lattices, respective Voronoi tessellations and hexagonality indices obtained for perturbation intensities $\delta=2$ and 4 , respectively. The histograms of the number of Voronoi cell sides, coefficient of variation of shortest distances, conformity ratio of shortest distances, shortest distance values, average angle difference (in degrees), and hexagonality indices for each of the perturbation intensities are shown in Figure 5

It is clear from these results that the number of Voronoi cell sides, shown in Fig. 6(a), is largely invariant to the perturbation intensity. In the cases where the latter is large enough to induce changes, it acts mainly by increasing the dispersion of the measurements while keeping an almost constant average value. The coefficient of variation (b), first shortest distance (d) and sum of angle differences (e) all resulted in an almost linear mapping between the perturbation intensity and the measurement. All these cases, and especially the coefficient of variation, are also characterized by an increase of dispersion for larger perturbations. The conformity ratio resulted the most non-linear relationship, providing greater sensitivity for smaller perturbations. However, the measurement dispersions are also substantially larger in such cases, which limits the discriminative power of this measurement. The hexagonality index, shown in Fig. 6](f), accounts for an almost linear mapping, with a slight increased sensitivity for small perturbations. In addition to these interesting features, this measurement is the only one characterized by an almost constant standard deviation which, combined with the almost linear mapping characterizing this measurement, implies a constant discriminative power along all the considered perturbation intensities. The linearity, sensitivity and discriminative power of the measurements of spatial order are summarized in Table II It follows from such results that the hexagonality index is particularly suitable in cases of higher spatial order, while the sum of angles provides an interesting alternative for analysis of less ordered sys- tems.

The properties of the number of sides, shortest distance and hexagonality index are confirmed by analysis of the histograms in Figure 6. which show the distribution of such measurements for several perturbation intensities.

\section{B. Biological Data}

In order to further evaluate the performance of the conformity ratio and sum of angle differences, these two measurements have been applied to characterize the regularity of spatial distribution of two types of cones in agouti photoreceptor mosaics. The choice of the sum of angles instead of the hexagonality index was motivated by the fact that such systems involve relatively high levels of disorder.

The agouti (Dasyprocta agout), is a hystricomorph rodent whose diurnal habit and well-developed visual streak yield an interesting model for comparative studies of the visual system (e.g. 2, 13 ). The organization of mosaics formed by short-wavelength-sensitive cones (S-cones) and middle- to long-wavelength-sensitive cones (M/L-cones) photoreceptors in the agouti's retina have been analyzed. For this purpose we used two polyclonal antibodies that have been shown to label S-cones (JH455) or M/L-cones (JH492) in a range of mammals, using immunocytochemical methods largely according to the procedures described elsewhere [1]. Using $40 \times$ oil immersion objective, 25 fields taken with $250 \times 250 \mu \mathrm{m}$ for $\mathrm{M} / \mathrm{L}$ cones and 23 fields with $500 \times 500 \mu \mathrm{m}$ for $\mathrm{S}$ cones were acquired along the vertical-dorsal axis of the retina with the aid of an optical microscope (Eclipse E600, Nikon, Japan) equipped with a high-resolution video camera (Nikon 4500). The raw images of the cones were captured at the level of the inner segments. For carrying out the analysis, $x$ and $y$ coordinates were identified by using the Scion Image Software (ScionCorp).

Figure 7 shows the average conformity ratios ( $x$-axis) and sum of angle differences ( $y$-axis) obtained for each of the considered photoreceptor areas. It is clear from this figure that the two cone populations are clearly separated by considering the sum of angle differences, while substantial overlap is observed for the conformity ratio. The obtained separation is even more definite than previous results obtained for the same data set by using the lacunarity measurement of translational invariance [5]. The fact that the conformity ratio allowed the characterization of higher variability of the $M$ cones suggests that additional insights can be obtained by using combinations of these two measurements.

In order to investigate the effect of using successive shortest distances from each point, conformity ratios and coefficient of variations were obtained considering the second to the fifth shortest distances. Discriminant analysis [7, 10] [19] was then applied in order to identify the contribution of each of such measurements to the overll 


\begin{tabular}{||l|l|l|l||}
\hline Measurement & linearity & sensitivity & discriminative power \\
\hline Number of sides & very smal & very low & very low \\
Coef. of variation & high & almost constant & higher for small perturbations \\
Conformity ratio & small & higher for small perturbations & higher for large perturbations \\
shortest distance & high & almost constant & higher for large perturbations \\
sum of angle diffs. & high & almost constant & higher for small perturbations \\
hexagonality index & medium & slightly higher for small perturbations & high and almost constant \\
\hline
\end{tabular}

TABLE I: The linearity, sensitivity and discriminative power of the considered measurements of spatial order.

separation between the two types of mosaics. The results indicated that the sum of angles is the only measurement among all possible linear combination of the considered features which allows all the mosaic types to be correctly identified. The use of conformity ratios and coefficients of variation for several shortest distances always implied in misclassifications of the mosaics.

\section{Characterization of Local Spatial Order}

Although we have so far concentrated on the characterization of the global properties of the spatial order in systems of points, another relevant problem involves the quantification of local order around each point. This issue is particularly important because, provided one can quantify the spatial order around a small neighborhood of each point, it becomes possible to study how such a measurement varies along the whole system of points. A particularly interesting example of such cases, which often appears in complex systems, is the existence of multiple spatial domains characterized by varying levels of spatial order. This situation is illustrated in Figure 9] including two simulated regions with different levels of spatial order. These domains, separated by an oblique border at the middle of the image, were obtained by perturbing a hexagonal lattice with $\delta=2$ and 4 , respectively. Such a relatively small variation of spatial order was intentionally imposed in order to impose a more demanding discrimination task on the measurements.

Another interesting situation is characterized by the progressive variation, along space, of the spatial order. A typical example of such systems is the spatial distribution of photoreceptors in the mammals retina. Higher spatial order, actually almost perfectly hexagonal, is observed for those receptors near the central part of the retina, becoming less and less ordered as one moves toward the retina periphery. Figure 10 illustrates this type of point system, derived from a hexagonal lattice by adding perturbation values increasing linearly with the distance from the center of the system of points.

Note that the conformity ratio and coefficient of variation can not be used for quantifying local order, as the standard deviation of the shortest distances for a single point becomes equal to zero. Therefore, we limit the subsequent analysis to the hexagonality index, the sum of angle differences, and the first to third shortest distances. Figure 8 shows the average \pm standard deviation considering all individual points in each of the 50 realizations of systems of points for each perturbation intensity. Except for the higher dispersions, these results are similar to those obtained by averaging globally over each realization shown in Figure 5

Figure 9 presents the results of local order identification of the domains in Figure ??(a) by using the first (a), second (b) and third (c) shortest distances as well as the hexagonality index (d). In order to obtain the results in Figure 9 the above measurements were obtained for each point and Bayesian decision theory (e.g. 6, 7] ) was then applied in order to obtain the best threshold for separating between the two groups. The points identified as belonging to the higher order group are represented as the squares with holes in Figure 9 Two particularly interesting results can be inferred from the obtained results: (i) the use of successive distances tend to reduce the correct identification of the regions and (ii) the hexagonality index provided the best overall classification of points. The former effect suggests further insights about the fact that the consideration of further shortest distances does not tend to contribute significantly to the characterization of global order (see Section IVB).

Figure 10 presents the quantification of the local spatial order of the simulated retina by using the shortest distance (a) and sum of difference angles (b). The enhanced sensitivity of the latter measurement is again corroborated by the more clearly defined gradient of hexagonality values as one moves radially from the center of the structure towards its border. The higher uniformity of the spatial order of the central cells is also more clearly identifiable by using the sum of angle differences.

\section{CONCLUDING REMARKS}

The important problem of quantifying the spatial order of systems of points has been addressed. A series of re- 


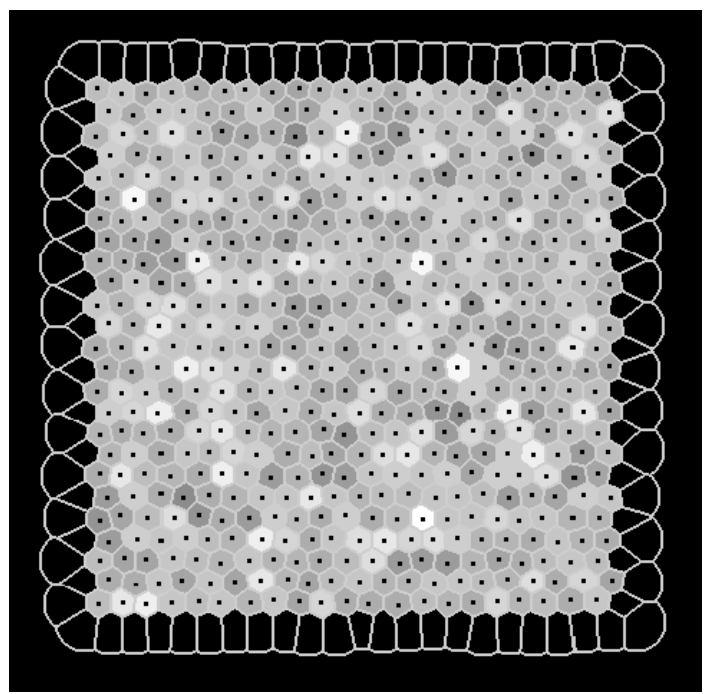

(a)

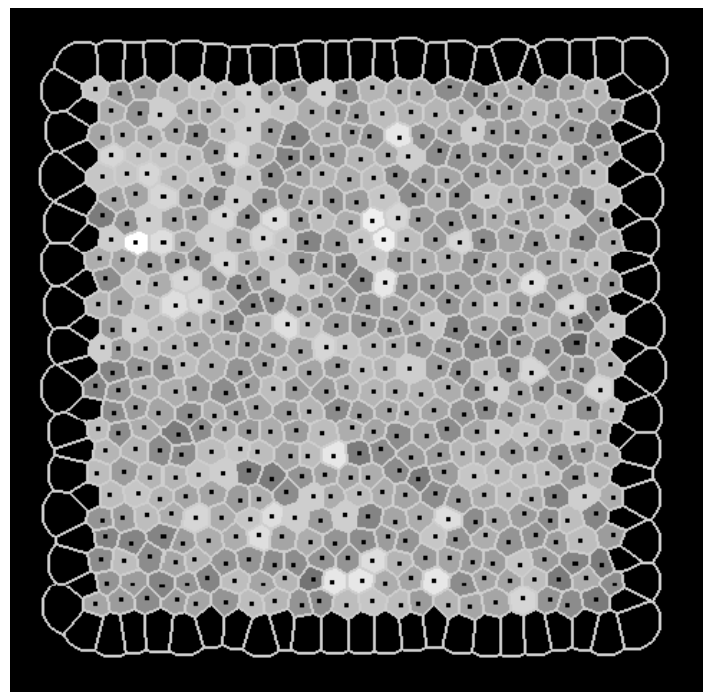

(b)

FIG. 4: Examples of simulated hexagonal lattices with perturbations $\delta=2$ (a) and 4 (b). The hexagonality index is expressed by the gray levels at each Voronoi cell, with higher values indicated in light gray. Note the border elements in black, whose hexagonality is not considered. The minimum and maximum gray levels in each image are normalized between black and white for the sake of better visualization.
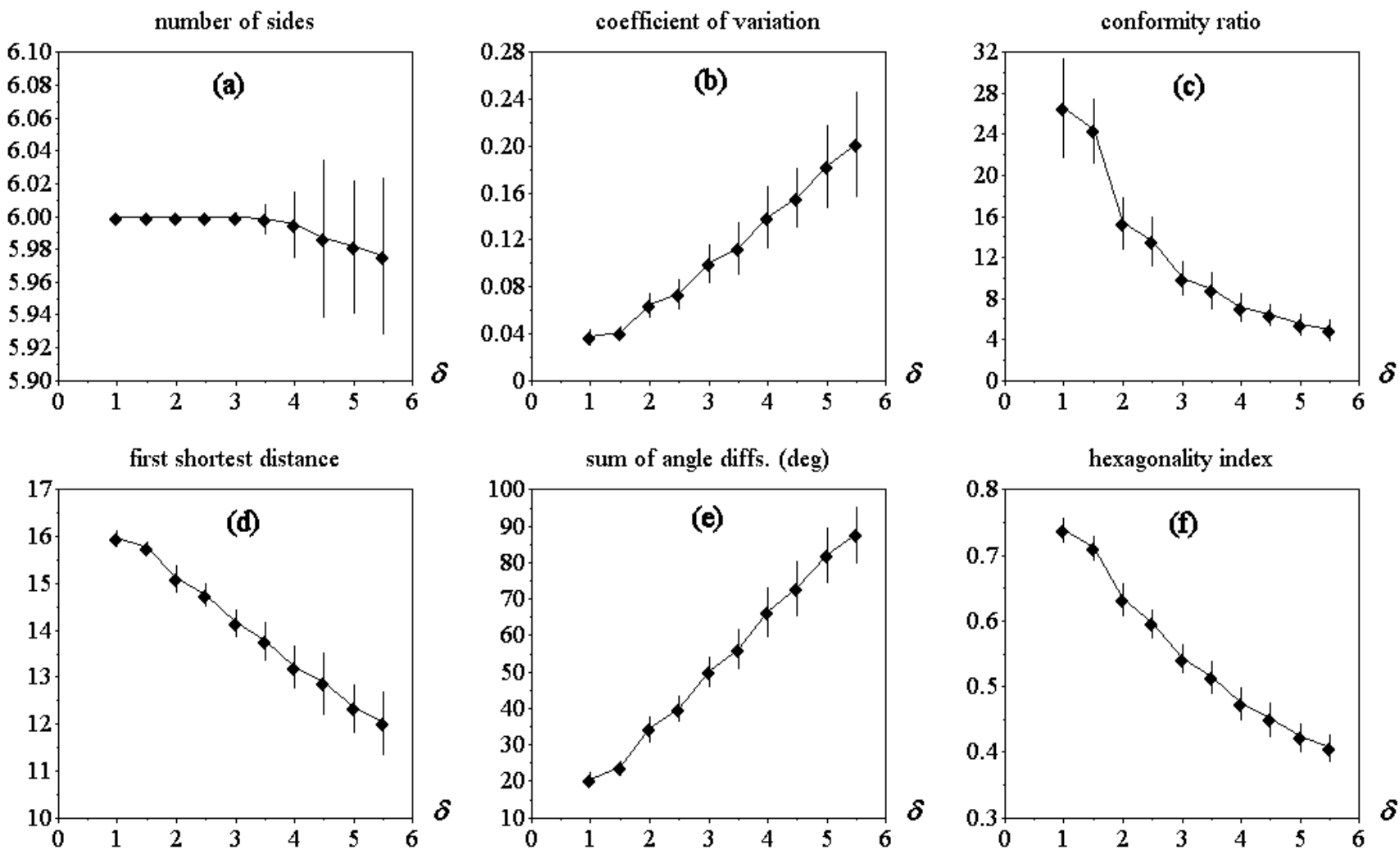

FIG. 5: The average \pm standard deviation of the number of Voronoi cell sides (a), coefficient of variation of first shortest distance (b), conformity ratio of first shortest distance (c) first shortest distances (d), average of angle differences (e), and hexagonality indices (f), in terms of the perturbation intensity $\delta$ considering all simulations. For the sake of better visualization, the standard deviations are shown to 5 times their original values. 
quirements expected from a good measurement of spatial order were identified, allowing the discussion and comparison of a series of traditional (number of neighbors, nearest distances between pairs of points, conformity ratio and coefficient of variation of nearest distances) as well as a recently introduced (the hexagonality index) and a novel (the sum of angle differences) measurements.

The potential of such measurements has been investigated with respect to the quantification of global and local spatial order. In the former case, which involves assigning a single measurement to the whole system of points in order to characterize its order, the measurements were first compared with respect to simulated data, namely hexagonal lattices with progressive perturbation intensities $\delta$. The obtained results allowed the objective characterization of the linearity, sensitivity and discriminative power of each considered measurement with respect to different levels of spatial order and hexagonality. The hexagonality index was verified to account for good linearity and slightly enhanced sensitivity for higher spatial orders, with a nearly constant discriminative power. The sum of angle differences also yielded interesting properties including good linearity and discriminative power. The identification of the type of retinal mosaics in the agouti was also considered as a practical problem involving the estimation of spatial order. Because such systems involve relatively low spatial order, the sum of angle differences was used instead of the hexagonality index. Conformity ratios and coefficient of variations involving the first to fifth nearest distances were also calculated and used as subsidy for distinguish- ing between the two types of mosaics. Discriminant analysis shown that the sum of angle differences was the only feature allowing perfect classification.

The possibility to use the considered measurements to quantify local spatial order was also addressed with respect to progressively perturbed hexagonal lattices, yielding similar results as for the global analysis, but with substantially higher dispersions of measurement values for each perturbation intensity. Two important applications of local order restimation, namely the identification of regions and analysis of systems of points involving gradients of spatial order, were also addressed. Such applications further corroborated the good features of the sum of angle differences and hexagonality index as estimators of spatial organization.

Such results imply that representative new information and insights about ordered biological systems can be obtained by revisiting previous investigations reported in the literature while considering a combination of sum of angle differences and hexagonality index measurements. Future works should include the extension of the angular polygonality measurements to higher dimensions and applications to the characterization of texture and gene expression patterns.

Luciano da F. Costa is grateful to FAPESP (process 99/12765-2), CNPq (308231/03-1) and the Human Frontier Science Program for financial support.
[1] P. K. Ahnelt, J. N. Hokoc, and P. Roehlich. Photoreceptors in a primitive mammal, the south american opossum, didelphis marsupialis aurita: Characterization with anti-opsin immunolabeling. Vis. Neurosc., 12:793-804, 1995.

[2] L. C. Silveira C., Picanço-Diniz, and E. Oswaldo-Cruz. Distribution and size of ganglion cells in the retinae of large Amazon rodents. Vis. Neurosc., 2(3):221-235, 1989.

[3] J. E. Cook. Spatial properties of retinal mosaics: An empirical evaluation of some existing measures. Vis. Neurosc., 13:15-30, 1996.

[4] L. da F. Costa, G. Riveros, H. Gómes, A. Cortes, M. Gilles, E. A. Dalchiele, and R. E. Marotti. Characterizing the hexagonality of anodic aluminium oxide nanoporous arrays. Appl. Phys. Lett. (submitted), 2005. cond-mat/0505185.

[5] L. da F. Costa, F. Rocha, and S. M. A. de Lima. Statistical mechanics characterization of neuronal mosaics. Appl. Phys. Lett., 86:093901, 2005. q-bio.NC/0411030.

[6] Luciano da F. Costa and Roberto Marcondes Cesar Jr. Shape Analysis and Classification: Theory and Practice. CRC Press, Boca Raton, 2001.

[7] R. O. Duda, P. E. Hart, and D. G. Stork. Pattern Classification. Wiley-Interscience, New York, 2001.

[8] P. V. Escribá, M. Sastre, and J. A. García-Sevilla. Disruption of cellular signaling pathways by daunomycin through destabilization of nonlamellar mebrane structures. Proc. Natl. Acad. Sci., 92:7595-7599, 1995.

[9] R. J. Freund and W. J. Wilson. Statistical Methods. Academic Press, 1993.

[10] J. F. Hair, R. E. Anderson, R. L. Tatham, and W. C. Black. Multivariate Data Analysis. Prentice Hall, 1998.

[11] O. Nishi and K. Hanasaki. Automated determination of polygonality of corneal endothelial cells. Cornea, 8(1):5457, 1989.

[12] A. Okabe, B. Boots, K. Sugihara, and S. N. Chiu. Spatial tessellations - Concepts and Applications of Voronoi Diagrams. John Wiley and Sons, 2000.

[13] C. Picanço-Diniz, L. C. Silveira, M. S. P. de Carvalho, and E. Oswaldo-Cruz. Contralateral visual field representation in area 17 of the cerebral cortex of the agouti: A comparison between cortical magnification factor and retinal ganglion cell distribution. Neurosc., 44:325-333, 1991.

[14] J. A. Pigatto, M. C. Andrade, J. L. Laus, J. M. Santos, D. E. Brooks, P. M. Gudes, and P. S. Barros. Morphometric analysis of the corneal endothelium of yacare caiman (Cayman yacare) using scanning electron microscopy. Vet. Ophtalmol., 7(3):205-208, 2004.

[15] R. L. Rockhill, T. Euler, and R. H. Masland. Spatial order within but not between types of retinal neurons. Proc. Natl. Acad. Soc., 29:2303-2307, 2000. 

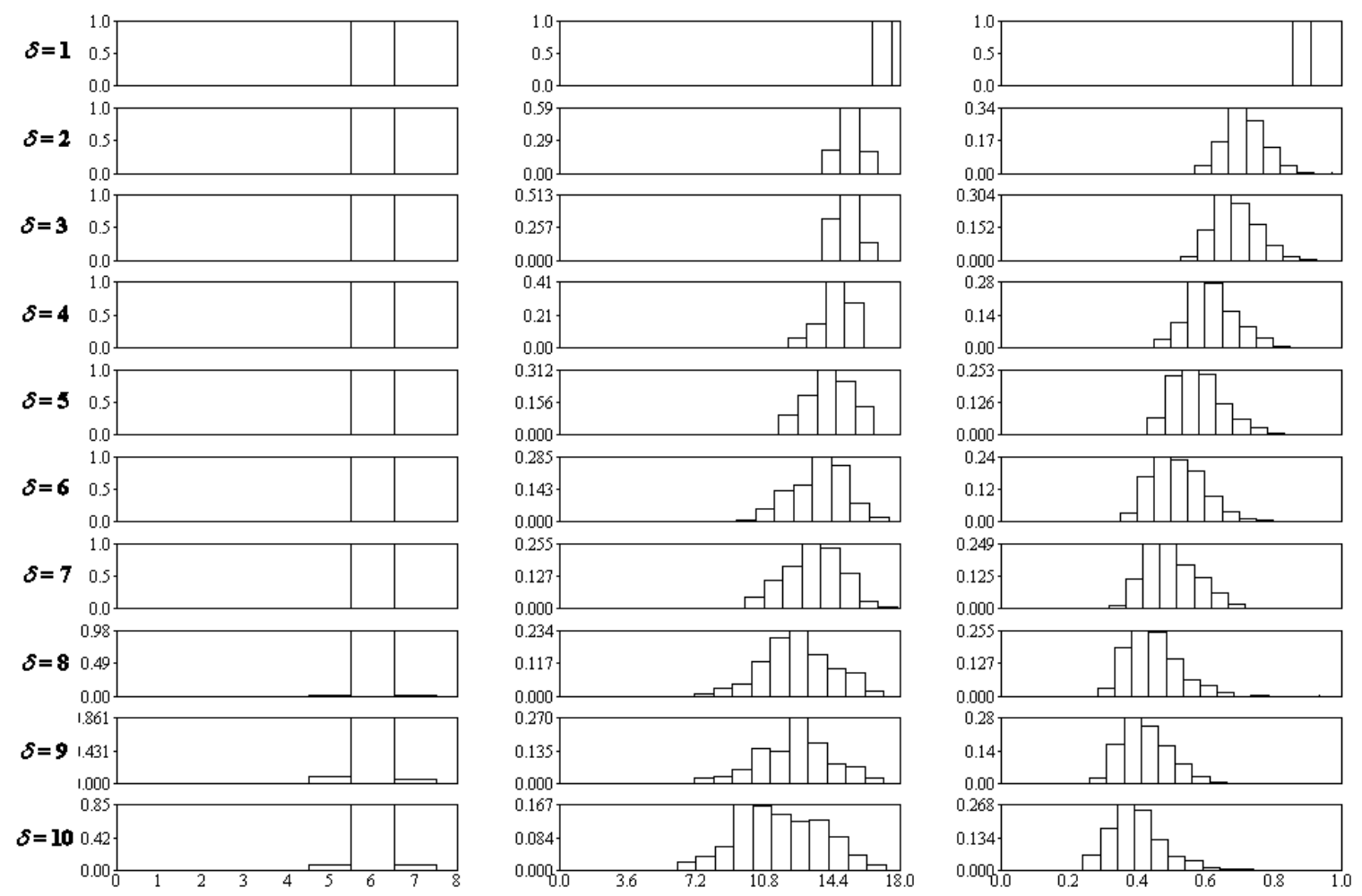

FIG. 6: The histograms of number of Voronoi cell sides (first column), nearest neighbor distances (second column) and hexagonality indices (third column) obtained for 50 realizations of each of the configuration, with progressive perturbation intensities $\delta$. 


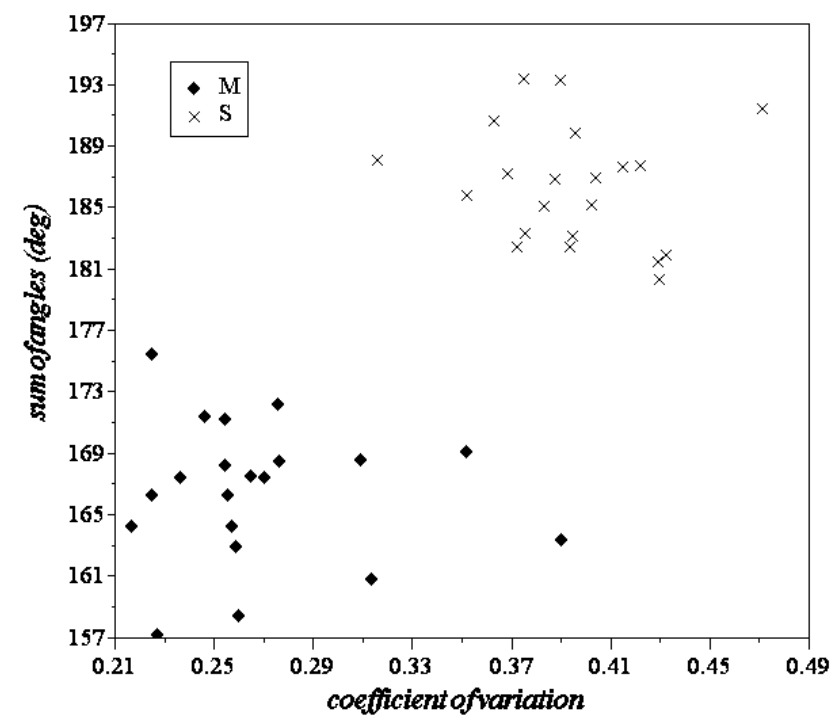

FIG. 7: Average conformity ratio and sum of angle differences obtained for each of the cone mosaics. Note that the $S$ and $M / L$ cone populations can be completely separated by considering the sum of angle differences isolately, which is not possible in the case of the conformity ratio. On the other hand, the conformity ratio allowed the identification of a high variability of the $M / L$ cone arrangements.
[16] H. Waessle and H. J. Riemann. The mosaic of nerve cells in the mammalian retina. Proc. Royal Soc. B (London), 200:441-461, 1978.

[17] J. M. Ziman. Models of disorder. Cambridge University Press, 1979.

[18] The property $P$ may also be a function of a series of parameters $\vec{s}$. The situation considered in this article assumes all but the single parameter $s$ are fixed.

[19] This type of statistical analysis involves the identification of the project from a high dimensional measurement space into a few dimensions so as to optimize the separation between the involved classes of objects. 

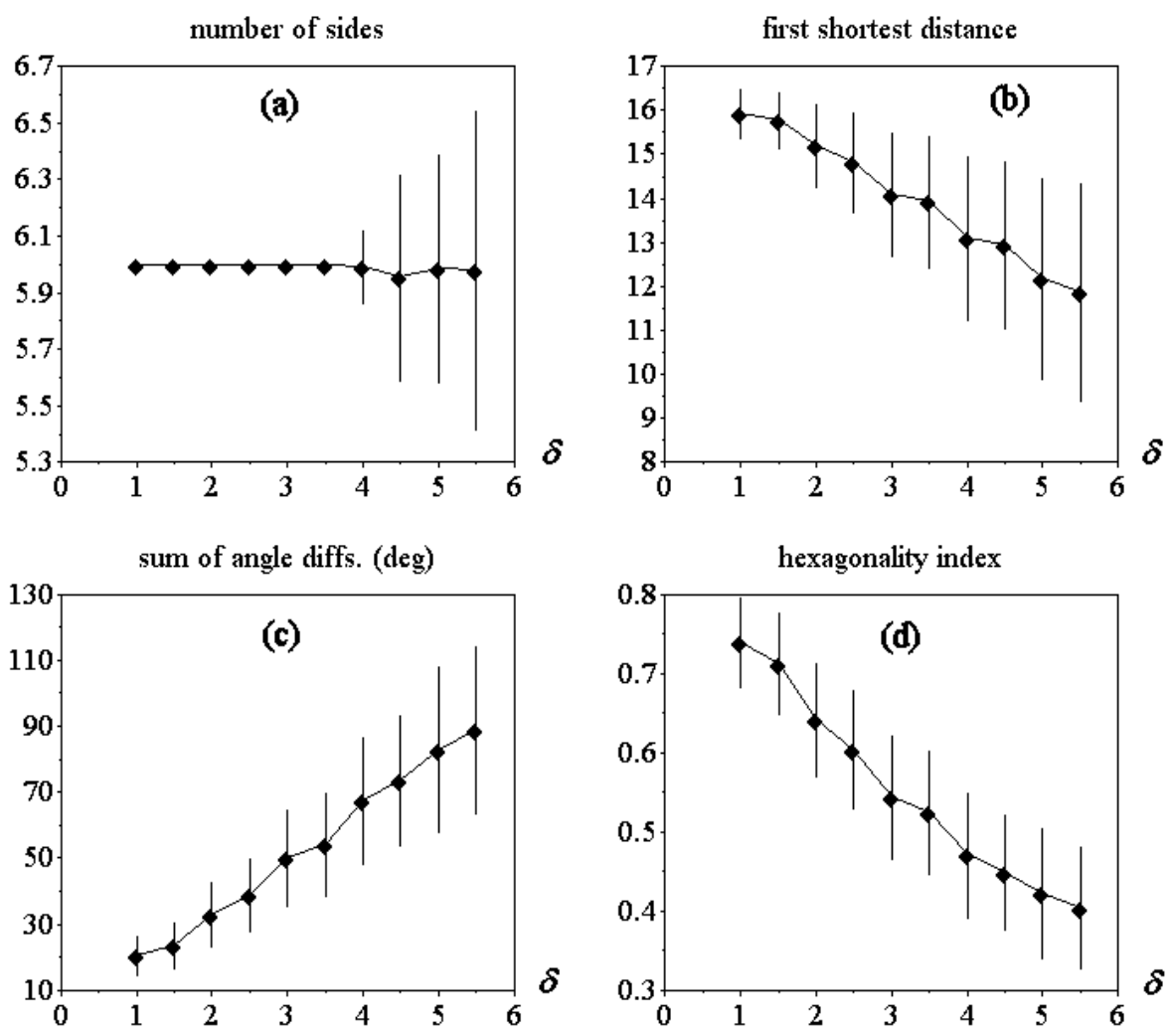

FIG. 8: The average \pm standard deviation of the number of Voronoi cell sides (a), first shortest distances (b), average of angle differences (c), and hexagonality indices (d), in terms of the perturbation intensity $\delta$ considering all individual points in the simulations. 


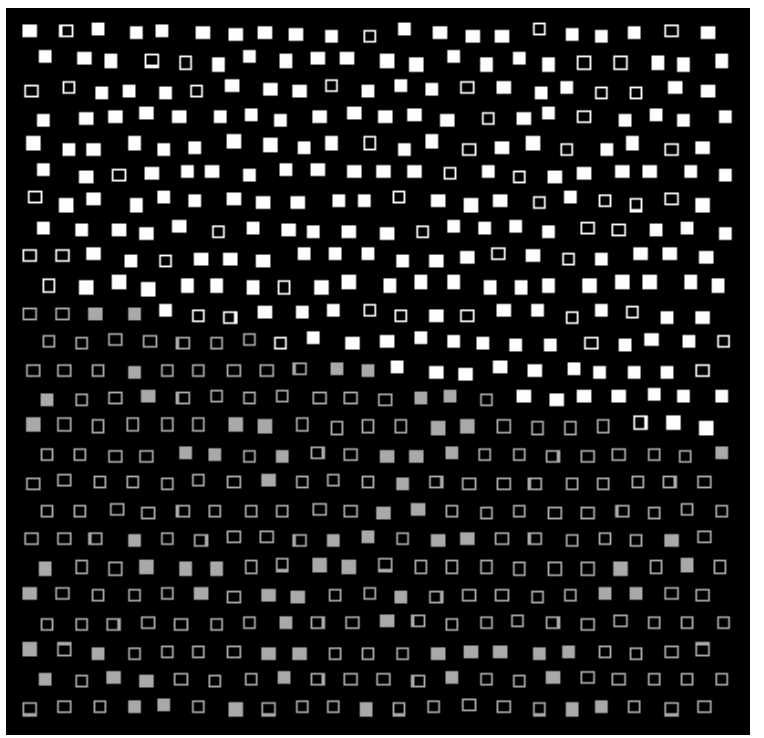

(a)

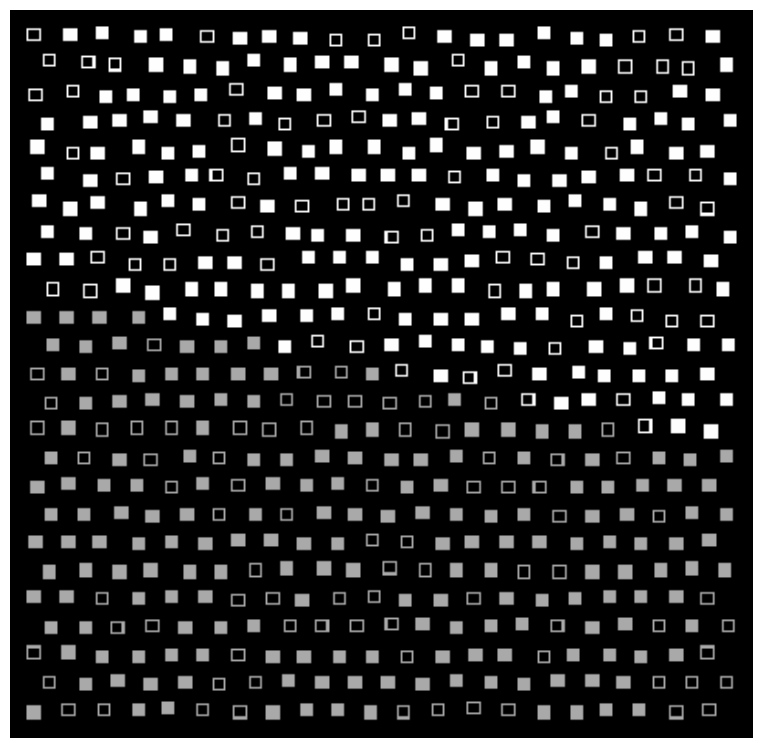

(c)

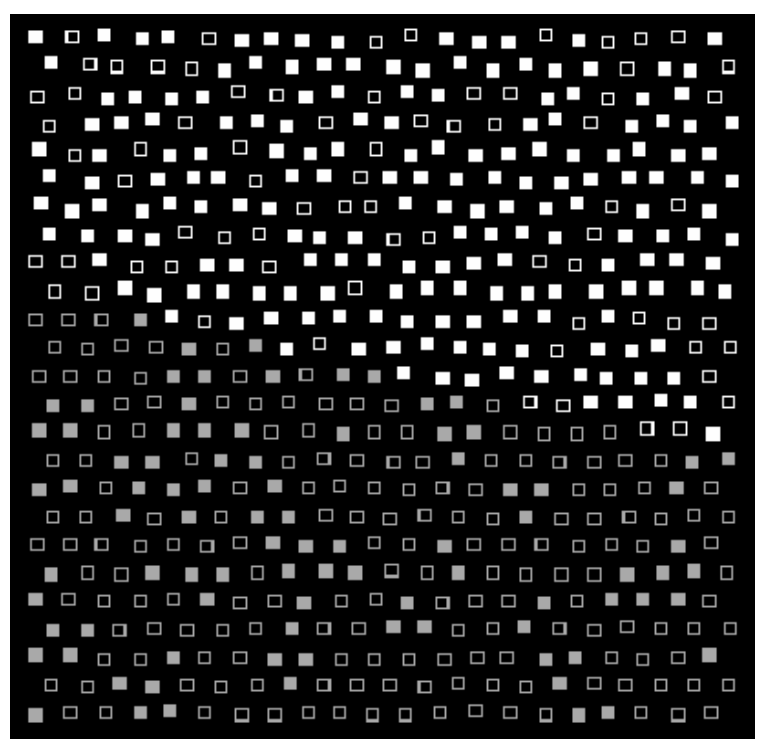

(b)

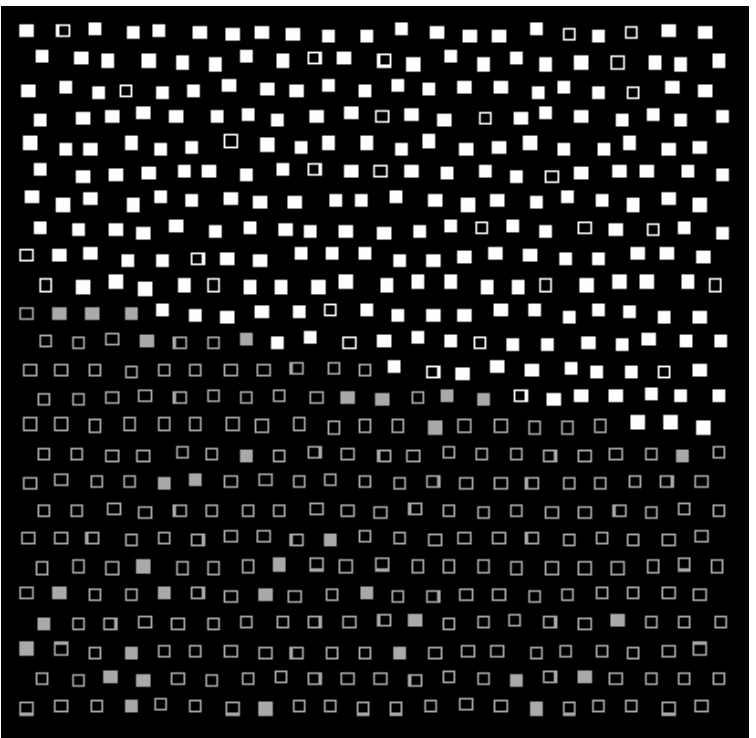

(d)

FIG. 9: Local order identification of a system of points involving two regions with distinct spatial order by using the first (a), second (b) and third (c) shortest distances and the hexagonality index (d). The squares with holes correspond to the points whose measurements were more likely to belong to the lower domain (higher spatial order.) 


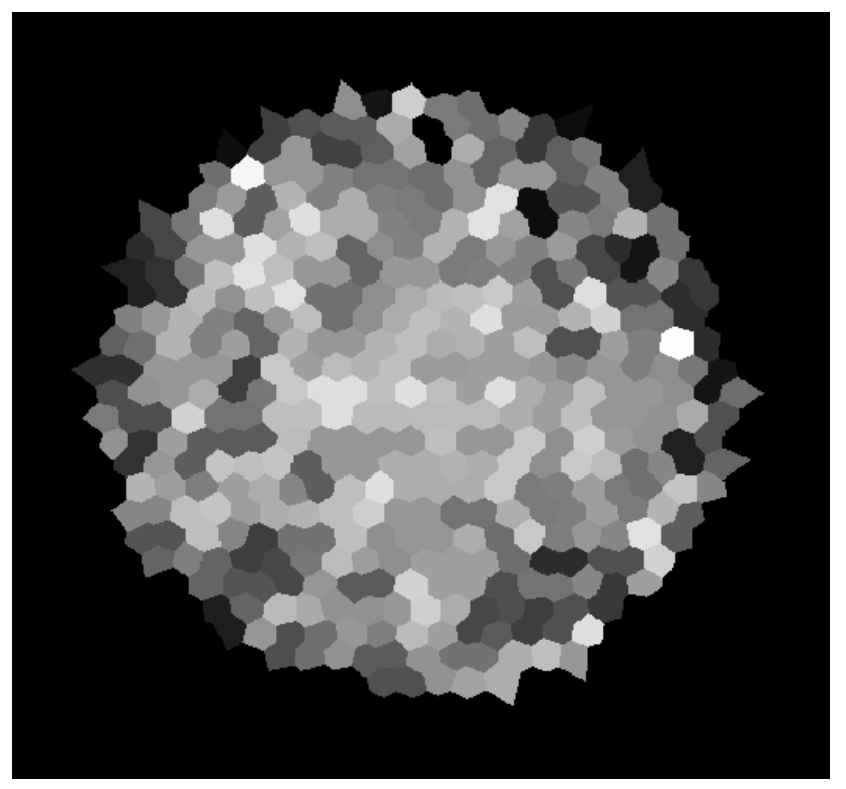

(a)

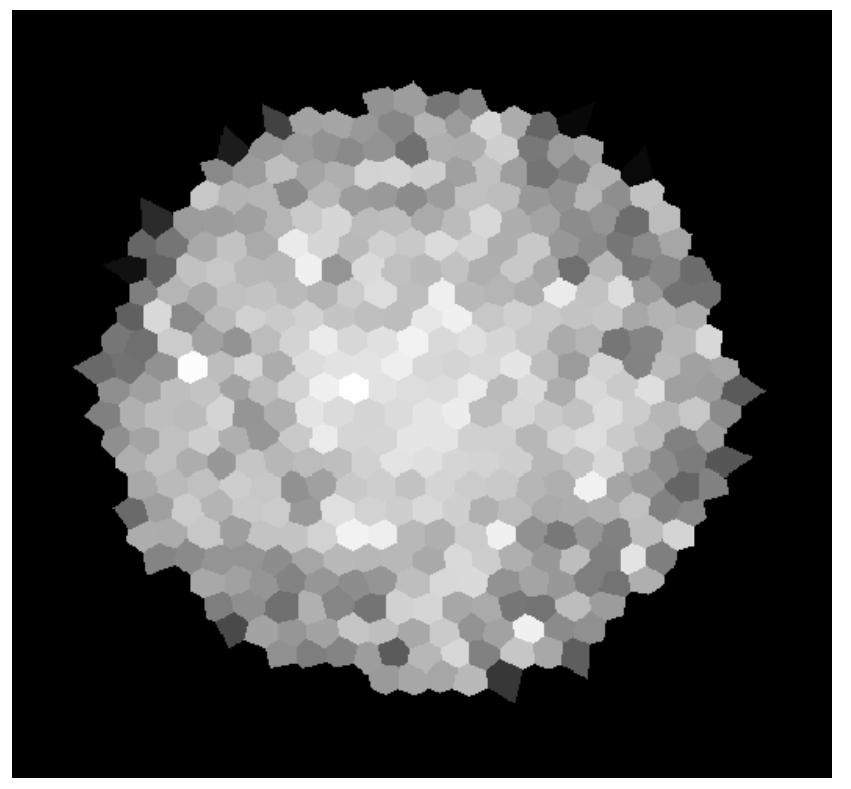

(b)

FIG. 10: Distribution of local order for the simulated retina by using the shortest distance (a) and the hexagonality index(b). The Voronoi cells belonging to the borders of the structure have been disconsidered. 


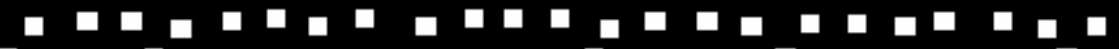

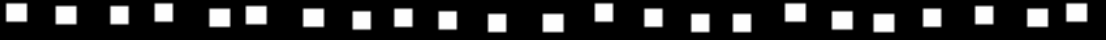

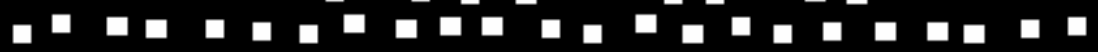

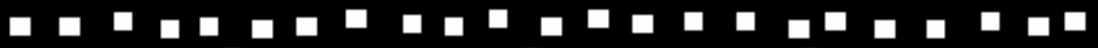

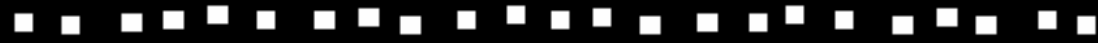

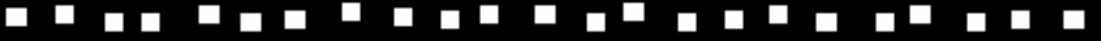

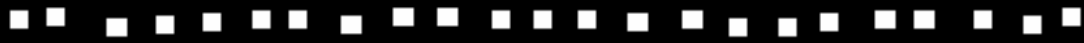
口 व

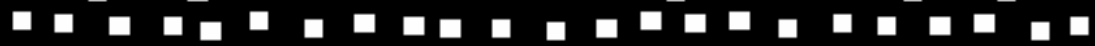

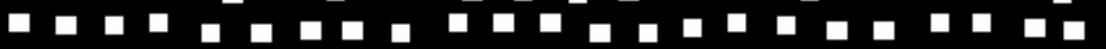

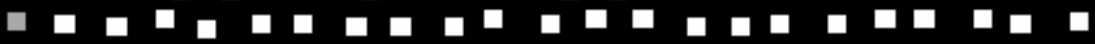
ㅁ

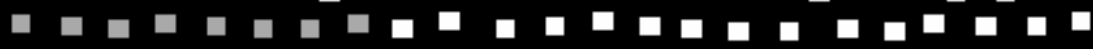
무

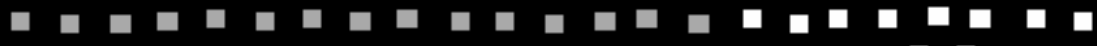
ㅁㅁㅁ

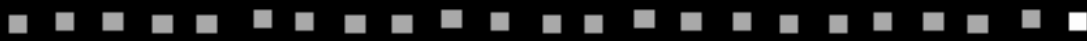
뭄ㅁ

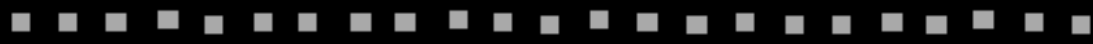

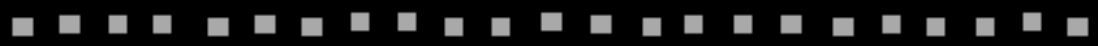

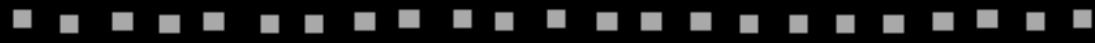

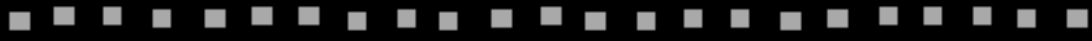
a 뭄

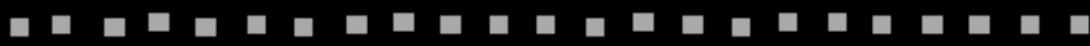

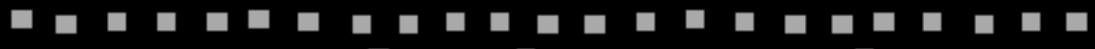

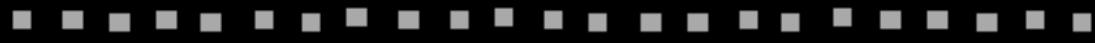




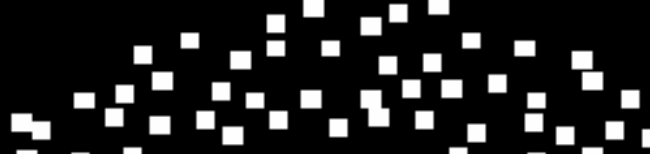

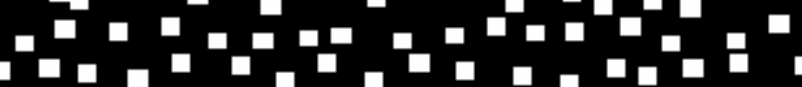
ת

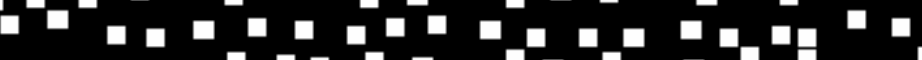

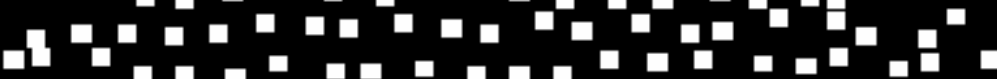
0.

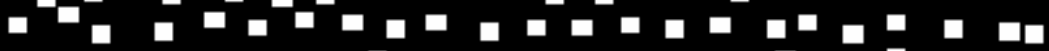
a

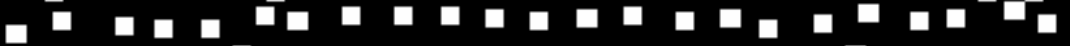

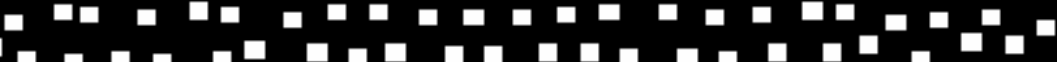

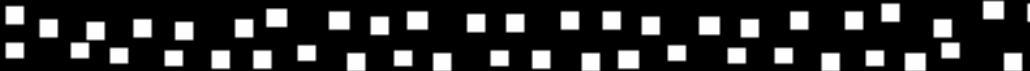

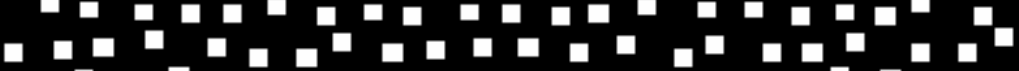

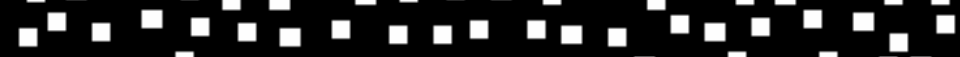

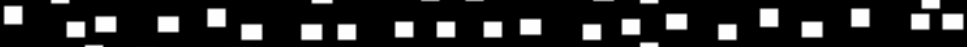
c

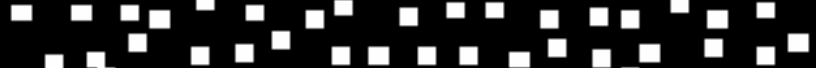
0 a

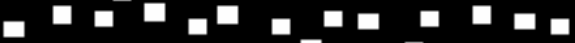
व प व 口ם $\square$ 